\title{
Rs2200733 and rs10033464 on chromosome 4q25 confer risk of cardioembolic stroke: an updated meta-analysis
}

\author{
Yan-yan Cao · Fei Ma $\cdot$ Yan Wang • \\ Dao Wen Wang $\cdot$ Hu Ding
}

Received: 19 March 2013/ Accepted: 14 September 2013/Published online: 25 September 2013

(C) The Author(s) 2013. This article is published with open access at Springerlink.com

\begin{abstract}
A recent genome-wide association study elucidated that $4 \mathrm{q} 25$ was implicated in ischemic stroke, but subsequent studies showed inconsistent results. In order to get coincident conclusion, we investigated two SNPs (rs2200733, rs10033464) on chromosome 4q25 in 1,388 stroke patients and 1,629 controls from Chinese Han population and then performed a meta-analysis. Although we failed to detect any association between $4 \mathrm{q} 25$ and stroke in our case-control study, meta-analysis revealed that rs2200733 showed association with overall stroke (OR $1.18,95 \%$ CI 1.08-1.27), but not for rs10033464. Subsequently subgroup analysis indicated that both rs2200733 and rs10033464 conferred increased risk for cardioembolic stroke (CE stroke) (for rs2200733, OR 1.38, $95 \%$ CI 1.26-1.51; for rs10033464, OR 1.14, $95 \%$ CI 1.02-1.26), while rs2200733 was marginal associated with non-CE
\end{abstract}

Yan-yan Cao and Fei Ma have contributed equally to this work.

Electronic supplementary material The online version of this article (doi:10.1007/s11033-013-2707-z) contains supplementary material, which is available to authorized users.

Y. Cao $\cdot$ F. Ma $\cdot$ Y. Wang $\cdot$ H. Ding $(\bowtie)$

Institute of Hypertension and Department of Internal Medicine, Tongji Hospital, Tongji Medical College, Huazhong University of Science and Technology, 1095\# Jiefang Ave, Wuhan 430030, People's Republic of China

e-mail: huding@tjh.tjmu.edu.cn

D. W. Wang $\cdot$ H. Ding

Genetic Diagnosis Center, Tongji Hospital, Tongji Medical

College, Huazhong University of Science and Technology,

Wuhan 430030, People's Republic of China

e-mail:dwwang@tjh.tjmu.edu.cn stroke (OR 1.09, $95 \%$ CI 1.02-1.16). our results demonstrated that two SNPs (rs2200733 and rs1003346) on chromosome $4 \mathrm{q} 25$ were limited to the stroke of cardioembolic etiology. To confirm this conclusion, welldesigned studies with larger sample size involving casecontrol populations with homogeneous ancestry warrant to be conducted in the future.

Keywords Stroke $\cdot 4 q 25 \cdot$ Meta-analysis

\section{Introduction}

Stroke is a multifactorial complex disorder causing longterm disability and increasing family responsibilities [1]. With high morbidity and mortality, stroke has become the first cause of death in China [2]. It was no doubt that environmental and clinical risk factors (such as hypertension, diabetes, hyperlipidemia, high body mass index (BMI) and smoking state etc.) contributed to the progress of stroke [3, 4], however twin and family history studies suggested that a genetic component also played an important role [5, 6]. Recently, a GWAS from Iceland found that rs2200733 and rs10033464 on chromosome $4 q 25$ both were strongly associated with stroke [7]. However, a series of later studies failed to replicate this result and obtained inconsistent results [8-15]. Aiming to get reliable and stable conclusion about the relationship between 4q25 and stroke, we analyzed two SNPs (rs2200733 and rs10033464) on chromosome 4q25 in Chinese Han population, including 1,388 stroke patients and 1,629 controls. Then, we combined our study with previously published articles to conduct a meta-analysis to asses the relationship between these two SNPs and the risk of overall stroke and subtypes. 


\section{Materials and methods}

Study subjects

This is a multicenter study sponsored by the Ministry of Science and Technology of China. Briefly, a total of 1,388 stroke patients were recruited in our study between November 2004 and January 2009 from five hospitals in Wuhan, China: atherothrombotic stroke (thrombosis, $n=716$ ), lacunar infarction (lacuna, $n=407$ ), and entracerebral hemorrhage (hemorrhage, $n=265$ ). The inclusion criteria and description about stroke subjects have been previously reported [16]. Ethnically and geographically matched controls $(n=1,629)$ were randomly selected from the healthy, community-based residents by house-to-house recruitment. All the study protocols were approved by the review board of the Ministry of Public Health, Ministry of Science and Technology of China and the ethics committees at all participating hospitals (Tongji Hospital, Union Hospital, Xinhua Hospital, First Wuhan Hospital and Wugang Hospital), and informed written consent was obtained from all participants. Experiments were conducted according to the principles expressed in the Declaration of Helsinki.

\section{DNA isolation and genotyping}

Genomic DNA was extracted using the QG-Mini80 workflow with a DB-S kit (FUJIFILM Corporation, Tokyo, Japan) as instructed. The SNPs were genotyped using the TaqMan SNP allelic discrimination on the TaqMan ${ }^{\mathrm{TM}}$ 7900HT Sequence Detection System under standard conditions as previously described [17]. Probe and primer sequences for these assays were custom designed by ABI Primer Expression 3.0 software and synthesized by GeneCore BioTechnologies Company, Limited, Shanghai, China (supplementary Table 1). Allelic discrimination was measured automatically using the Sequence Detection Systems 2.1 software (autocaller confidence level $95 \%$ ). A total of $10 \%$ of all genotypes were repeated in independent PCRs to check for consistency and to ensure intraplate and interplate genotype quality control. No genotyping discrepancies were detected between the repeated samples. In addition, all the DNA samples for cases and controls were run in the same batch. In order to verify the accuracy of Taqman probe method for genotyping, we randomly selected 151 (about $5 \%$ of sample size) samples from cases and controls to conduct direct DNA sequence analysis using the BigDye ${ }^{\circledR}$ Terminator v3.1 Cycle Sequencing Kits on an ABI PRISM 3130xl Genetic Analyzer (Applied Biosystems, Foster City, CA, USA). At last, we found these two different genotyping methods were highly consistent.
Statistical analysis

All quantitative variables were generally described as means with standard deviation (SD). one-way ANOVA test was performed to compare the baseline characteristics of different groups, such as age, BMI etc.; $\chi^{2}$ test was used for qualitative variables. Multiple unconditional logistic regression was used to estimate odds ratio (OR) and $95 \%$ confidence interval (CI) under different genetic models after adjusting for gender, age, BMI, hypertension, diabetes, hyperlipidemia and smoking status. Haplotype frequencies for these two SNPs combinations were first estimated by haplo.stats (version 12.1) for the $\mathrm{R}$ statistical package and then verified using Haploview 4.0. Both of the software above uses the expectation-maximization (EM) algorithm when constructing the haplotypes. Global score tests were applied to evaluate overall haplotype frequency differences between cases and controls, whereas the haplotype-specific score tests were performed to test individual haplotype difference between cases and controls. To minimize the falsepositive results generated from multiple statistical testing in our aforementioned analyses, we adopted the Bonferroni correction method for multiple testing.

\section{Literature search}

We systematically searched in Pubmed, Embase and CNKI up until December, 2012 using the key words "stroke", "ischemic stroke", "cerebral infarction", "cerebrovascular disease" paired with "4q25", "SNP", "PITX2", "rs2200733" and "rs10033464", respectively. Reference lists of relevant articles were also screened. The study inclusion criteria were as follows: (1) articles published in English or Chinese, (2) casecontrol or population-based studies, (3) studies with complete data on allele frequency and relevant clinical base characteristics, (4) studies about the association between rs2200733 or rs10033464 and stroke. Exclusion criteria were: (1) overlapping data, (2) case-only study, (3) small number cases, (4) review, (5) uncompleted article.

\section{Data extraction}

Two investigators independently extracted useful data from the articles meeting the inclusion criteria, including first author name, publication year, number of stroke patients and controls, ORs (HRs) and $95 \%$ CI. Disagreements were resolved by discussion.

Statistical meta-analysis

All meta-analyses were performed by STATA software (version10.0). Pooled OR and $95 \%$ CI were used to assess the association between rs2200733 or 10033464 and stroke. 
Table 1 Baseline characteristics of stroke samples

\begin{tabular}{llllll}
\hline Characteristics & $\begin{array}{l}\text { Controls } \\
n=1,629\end{array}$ & $\begin{array}{l}\text { Cases } \\
n=1,388\end{array}$ & $\begin{array}{l}\text { Thrombosis } \\
n=716\end{array}$ & $\begin{array}{l}\text { Lacunar } \\
n=407\end{array}$ & $\begin{array}{l}\text { Hemorrage } \\
n=265\end{array}$ \\
\hline Age (years) & $59.29 \pm 10.1$ & $61.2 \pm 10.4^{\mathrm{a}}$ & $60.9 \pm 10.4^{\mathrm{a}}$ & $64.4 \pm 9.0^{\mathrm{a}}$ & $57.14 \pm 11.0^{\mathrm{a}}$ \\
Male (\%) & 43.2 & $66.1^{\mathrm{a}}$ & $66.9^{\mathrm{a}}$ & $64.6^{\mathrm{a}}$ & $66.6^{\mathrm{a}}$ \\
BMI (kg/m $\left.{ }^{2}\right)$ & $24.2 \pm 3.4$ & $24.2 \pm 3.3$ & $24.3 \pm 3.2$ & $24.3 \pm 3.5$ & $23.8 \pm 3.5$ \\
SBP (mmHg) & $130.0 \pm 20.7$ & $148.3 \pm 23.0^{\mathrm{a}}$ & $147.6 \pm 24.1^{\mathrm{a}}$ & $145.4 \pm 21.9^{\mathrm{a}}$ & $154.5 \pm 25.5^{\mathrm{a}}$ \\
DBP (mmHg) & $80.3 \pm 22.3$ & $87.1 \pm 13.8^{\mathrm{a}}$ & $86.4 \pm 14.6^{\mathrm{a}}$ & $84.4 \pm 12.6^{\mathrm{a}}$ & $93.2 \pm 16.7^{\mathrm{a}}$ \\
Hypertension (\%) & 28.1 & $68.7^{\mathrm{a}}$ & $69.4^{\mathrm{a}}$ & $68.8^{\mathrm{a}}$ & $66.6^{\mathrm{a}}$ \\
Diabetes (\%) & 6.4 & $15.6^{\mathrm{a}}$ & $18.9^{\mathrm{a}}$ & $16.5^{\mathrm{a}}$ & \\
Hyperlipidemia (\%) & 23.6 & 25.2 & 29.3 & 27.5 & 5.3 \\
Smokers (\%) & 27.1 & $51.1^{\mathrm{a}}$ & $53.8^{\mathrm{a}}$ & $50.6^{\mathrm{a}}$ &
\end{tabular}

Values are expressed as mean \pm SD unless otherwise noted

$B M I$ body mass index, $S B P$ systolic blood pressure, $D B P$ diastolic blood pressure

a Test for differences between cases and controls, $P<0.01$

Table 2 Associations of SNPs with overall stroke and subtypes in different genetic models

\begin{tabular}{lllllllll}
\hline SNPs & Subtypes & $\begin{array}{l}\text { Minor allele frequency } \\
\text { Control/case }\end{array}$ & $\begin{array}{l}\text { ORs (95 \% CI) } \\
\text { Additive model }\end{array}$ & $P$ & $\begin{array}{l}\text { ORs (95 \% CI) } \\
\text { Dominant model }\end{array}$ & $P$ & $\begin{array}{l}\text { ORs (95 \% CI) } \\
\text { Recessive model }\end{array}$ & $\begin{array}{l}P \\
\text { rs2200733 }\end{array}$ \\
& Thrombosis & $0.473 / 0.467$ & $1.03(0.89-1.18)$ & 0.705 & $1.00(0.80-1.25)$ & 0.998 & $1.08(0.85-1.38)$ & 0.518 \\
& Lacuna & $0.473 / 0.456$ & $0.96(0.81-1.14)$ & 0.618 & $1.10(0.84-1.44)$ & 0.485 & $0.78(0.57-1.05)$ & 0.100 \\
& Hemorrhage & $0.473 / 0.458$ & $0.98(0.80-1.20)$ & 0.817 & $1.08(0.78-1.49)$ & 0.636 & $0.85(0.59-1.21)$ & 0.358 \\
& All & $0.473 / 0.462$ & $1.00(0.89-1.13)$ & 0.982 & $1.05(0.87-1.26)$ & 0.613 & $0.95(0.78-1.13)$ & 0.606 \\
rs10033464 & Thrombosis & $0.209 / 0.207$ & $1.05(0.88-1.25)$ & 0.612 & $1.09(0.88-1.35)$ & 0.412 & $0.89(0.55-1.45)$ & 0.636 \\
& Lacuna & $0.209 / 0.204$ & $1.01(0.82-1.25)$ & 0.893 & $1.07(0.83-1.38)$ & 0.599 & $0.76(0.40-1.43)$ & 0.380 \\
& Hemorrhage & $0.209 / 0.236$ & $1.29(1.02-1.64)$ & 0.038 & $1.47(1.08-1.97)$ & 0.015 & $1.11(0.59-2.07)$ & 0.748 \\
& All & $0.209 / 0.212$ & $1.06(0.92-1.22)$ & 0.445 & $1.11(0.93-1.32)$ & 0.237 & $0.89(0.60-1.32)$ & 0.556 \\
\hline
\end{tabular}

ORs and $P$ value were estimated by multiple unconditional logistic regression after adjusting for gender, age, body mass index, hypertension, diabetes, hyperlipidemia and smoking status

Heterogeneity was calculated by Cochran's Q statistic and inconsistency index $\left(\mathrm{I}^{2}\right)$. If probability values $<0.10$ or $\mathrm{I}^{2}>0.50$, heterogeneity was considered [18]; the random effects model was selected to estimate the pooled OR. Otherwise fixed effects model was applied. In order to reduce the heterogeneity of the meta-analysis, then, we performed subgroup analysis. Sensitivity analyses were used by omitting one cohort or one study at one time and calculating the pooled ORs of remaining cohorts or studies to assess the stability of our results. To test the publication bias, Begg' funnel plot and Egger' test were operated, All $P$ values were two-tailed and $P<0.05$ was considered statistically significant.

\section{Results}

Allelic association of SNPs in $4 \mathrm{q} 25$ and stroke

The characteristics of our study cohorts were shown in Table 1. Rs2200733 and rs10033464 genotypes were found to be in Hardy-Weinberg equilibrium in controls (both $P>0.05)$. Multivariate unconditional logistic regression analyses revealed that rs10033464 conferred risk for intracerebral hemorrhage in additive model (OR 1.29, $95 \%$ CI 1.02-1.24, $P=0.038$ ) and dominant model (OR $1.47,95 \%$ CI $1.08-1.97, P=0.015)$; rs 2200733 was not associated with overall stoke and subtypes (Table 2). However, none of associations remained significant with adjustment of Bonferroni correction.

Haplotype analysis for these two SNPs

The correlation of two SNPs (rs2200733 and rs10033464) within the region of $4 \mathrm{q} 25$ is weak in our control data set $\left(\mathrm{D}^{\prime}=0.91, \mathrm{r}^{2}=0.24\right)$. We used the haplo.stats program to determine whether the combined effects of two SNPs (rs2200733 and rs10033464) were associated with overall stroke and subtypes. As shown in Table 3, we failed to detect a significant difference for these two SNPs for all the 
Table 3 Association of haplotypes with overall stroke and subtypes

\begin{tabular}{|c|c|c|c|c|c|c|c|c|c|}
\hline Haplotype $^{\mathrm{a}}$ & Control & All stroke & $P^{\mathrm{b}}$ & Thrombosis & $P^{\mathrm{b}}$ & Lacunar & $P^{\mathrm{b}}$ & Hemorrhage & $P^{\mathrm{b}}$ \\
\hline $\mathrm{TG}^{\mathrm{c}}$ & 0.517 & 0.525 & 0.478 & 0.519 & 0.834 & 0.539 & 0.297 & 0.517 & 0.753 \\
\hline CG & 0.275 & 0.265 & 0.36 & 0.275 & 0.927 & 0.275 & 0.388 & 0.248 & 0.175 \\
\hline \multirow[t]{2}{*}{$\mathrm{CT}$} & 0.199 & 0.197 & 0.938 & 0.191 & 0.653 & 0.197 & 0.876 & 0.212 & 0.424 \\
\hline & & \multicolumn{2}{|c|}{ Global $P=0.680$} & \multicolumn{2}{|c|}{ Global $P=0.680$} & \multicolumn{2}{|c|}{ Global $P=0.601$} & \multicolumn{2}{|c|}{ Global $P=0.345$} \\
\hline
\end{tabular}

${ }^{a}$ Haplotype frequencies were inferred using the EM algorithm within the haplo.stats R package; haplotypes are not listed if all the estimated frequencies are $<0.02$ in controls, patients with stroke

b $P$ value based on haplotype-specific score tests

${ }^{c}$ Constituted by SNPs rs2200733 and rs10033464

Fig. 1 Flow chart of the this meta-analysis selection of articles included in

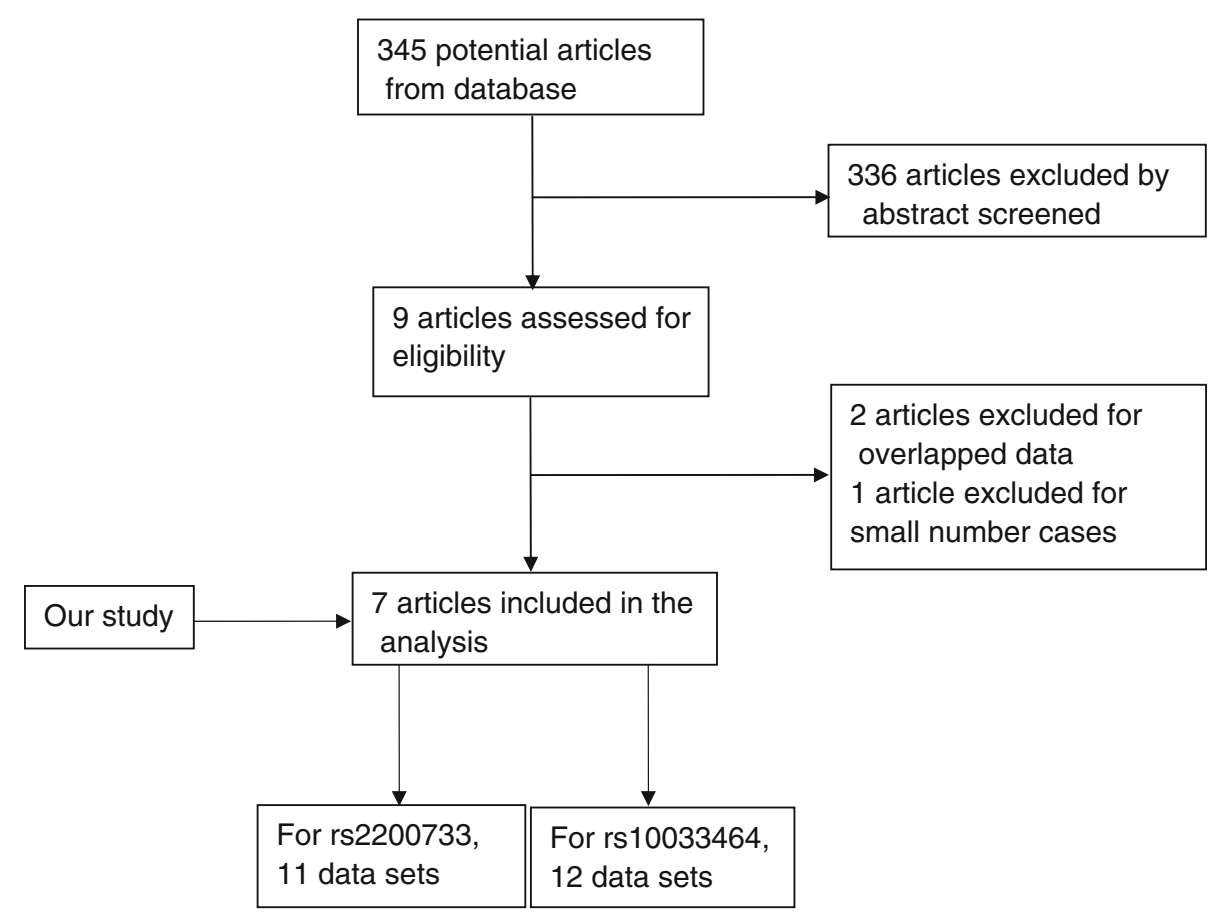

subjects at haplotype levels, which was consistent with single SNP analysis.

\section{Meta-analysis}

Data extraction process

We initially obtained 345 potential articles, among which most were excluded for no relevance to our analysis after screening abstract. Nine articles remained to assess the fulltext. Three articles then were removed because overlapping data $[10,13]$ and small number cases [14]. Finally, seven articles (combining our study) including 11 data sets for rs2200733 and 12 data sets for 10033464 met the inclusion criteria (Fig. 1).
Association of rs2200733 polymorphism with the risk of stroke

A total of 11 data sets from six studies (including our study) for rs2200733 polymorphism were involved in our meta-analysis containing 13,764 cases and 73,527 controls. The characteristics of these studies were shown in Table 4. In detail, seven data sets containing 3,825 cases and 40,980 controls described the association for CE stroke, as well as eight data sets $(9,939$ cases and 60,486 controls) for nonCE stroke. As shown in Fig. 2a, a random-effects model was performed for meta-analysis and generated a combined allelic OR of 1.18 for risk allele (95\% CI, 1.08-1.27) with heterogeneity $\left(\mathrm{Q}=29.67, \mathrm{I}^{2}=66.3 \%\right)$. When restricted to $\mathrm{CE}$ stroke subgroup studies, there was significant association between rs2200733 and the risk of CE stroke (OR 


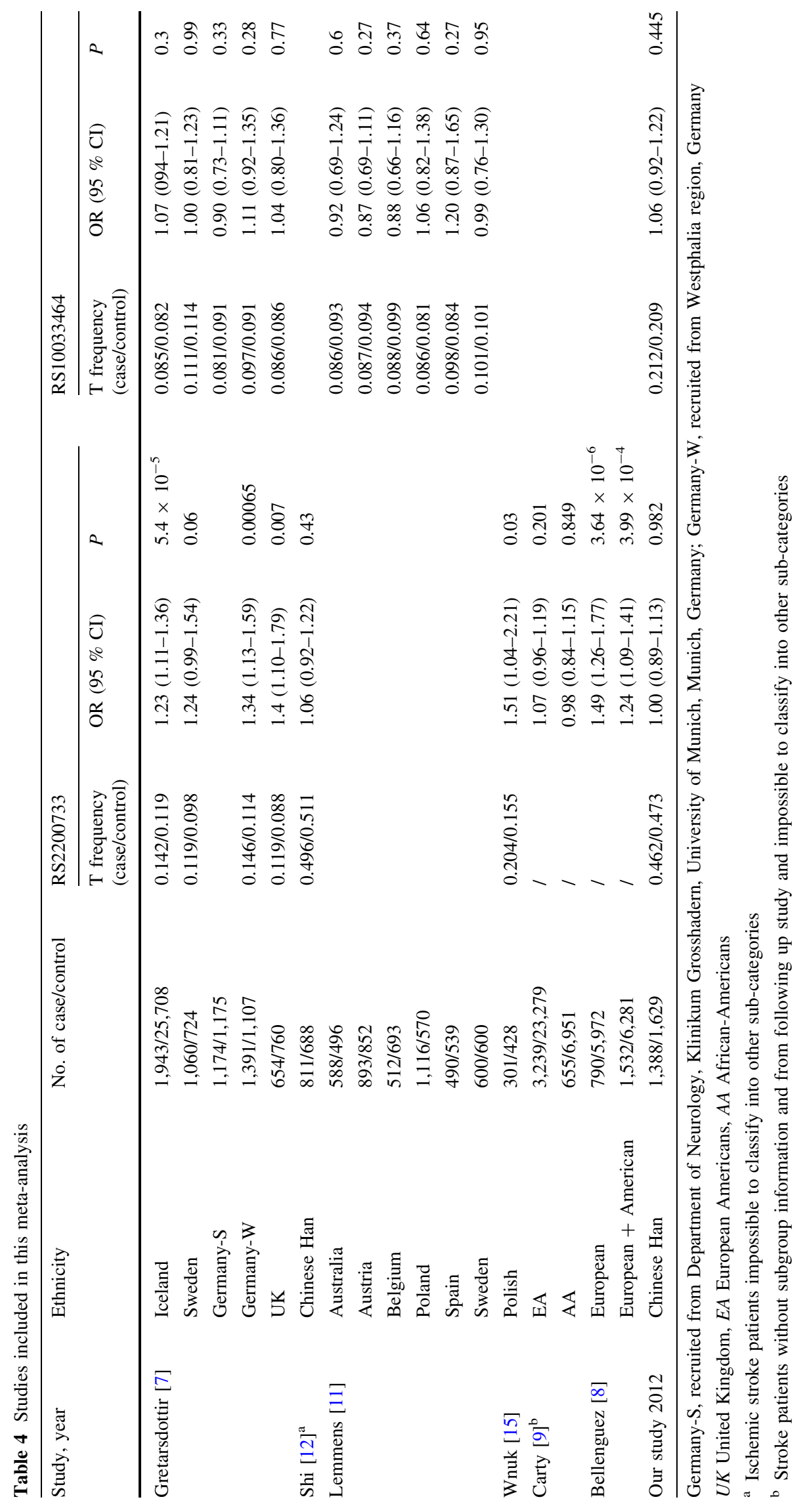


Fig. 2 Meta-analysis of the association between rs2200733 and overall stroke, CE stroke and non-CE stroke. The squares and horizontal lines refer to the study-specific OR and $95 \%$ CI. a Meta-analysis plot of association between rs2200733 and overall stroke. b Metaanalysis plot of association between rs2200733 and CE stroke. c Meta-analysis plot of association between rs2200733 and non-CE stroke (a) study

Gretarsdottir 2008

Gretarsdottir 2008

Gretarsdottir 2008

Gretarsdottir 2008

Shi 2009

Wnuk2011

Carty 2012

Carty 2012

Bellenguez 2012

Bellenguez2012

our study 2012

Overall (l-squared $=66.3 \%, p=0.001$ )

NOTE: Weights are from random effects analysis

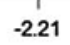

OR $(95 \% \mathrm{Cl})$

Weight \%

(b) Study

Gretarsdottir 2008

Gretarsdottir 2008

Gretarsdottir 2008

Gretarsdottir 2008

Wnuk 2011

Bellenguez 2012

Bellenguez 2012

Overall (1-squared $=9.7 \%, p=0.355$ )

NOTE: Weights are from random effects analysis

\begin{tabular}{ll|l}
\hline & & \\
\hline & 1 & 1
\end{tabular}

(c) study

OR $(95 \% \mathrm{Cl}) \quad$ Welght $\%$

Gretarsdottir 2008

Gretarsdottir 2008

Gretarsdottir 2008

Gretarsdottir 2008

Shi 2009

Carty 2012

Carty 2012

our study 2012

Overall (l-squared $=35.5 \%, p=0.145$ )

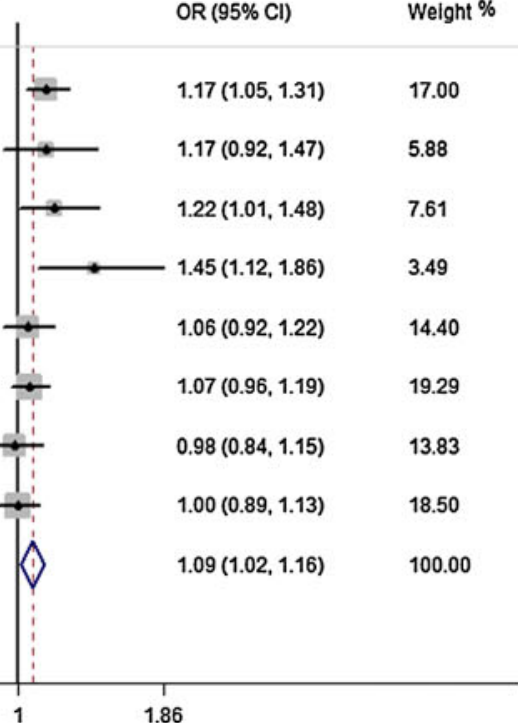


Fig. 3 Meta-analysis of the association between rs10033464 and overall stroke, CE stroke and non-CE stroke. The squares and horizontal lines refer to the study-specific OR and $95 \%$ CI. a Meta-analysis plot of association between rs10033464 and overall stroke. b Metaanalysis plot of association between rs10033464 and CE stroke. c Meta-analysis plot of association between rs10033464 and non-CE stroke

\section{(a) Study}

OR $(95 \% \mathrm{Cl})$

Weight\%

\section{Gretarsdottir 2008 \\ Gretarsdottir 2008 \\ Gretarsdottir 2008 \\ Gretarsdottir 2008 \\ Gretarsdottir 2008 \\ Lemmens 2010 \\ Lemmens 2010 \\ Lemmens 2010 \\ Lemmens 2010 \\ Lemmens 2010 \\ Lemmens 2010 \\ our study 2012}

Overall (1-squared $=0.0 \%, p=0.703$ )

NOTE: Weights are from random effects analysis

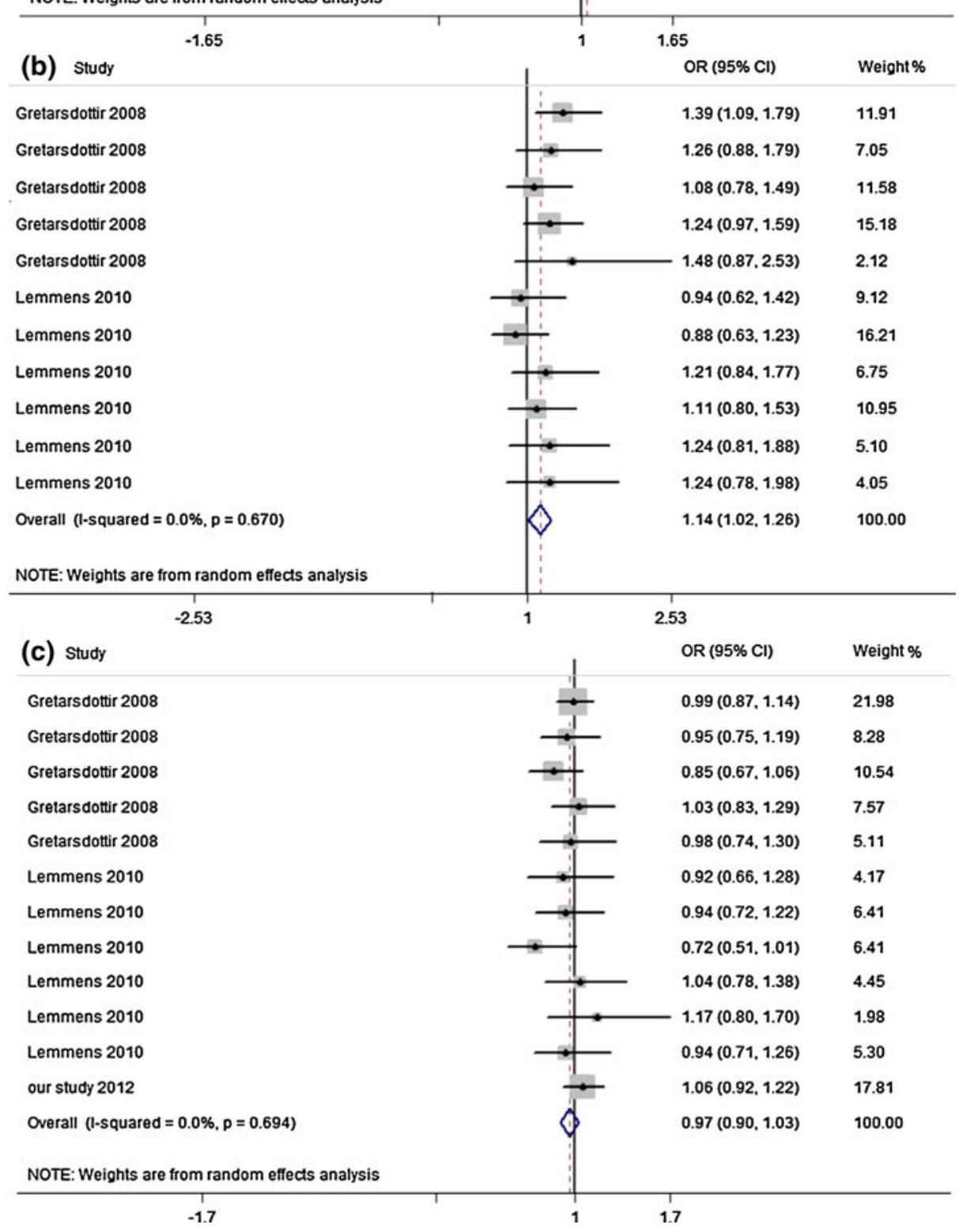


1.38, $95 \%$ CI 1.26-1.51) (Fig. 2b) and no obvious heterogeneity was found $\left(\mathrm{Q}=6.64, \mathrm{I}^{2}=9.7 \%\right)$. Figure $2 \mathrm{c}$ showed the association between rs2200733 and non-CE stroke (OR 1.09, $95 \%$ CI 1.02-1.16) was marginal and the heterogeneity of this meta-analysis reduced $(\mathrm{Q}=10.86$, $\left.\mathrm{I}^{2}=35.5 \%\right)$.

Association of rs10033464 and the risk of stroke

For rs10033464, 12 data sets (including our study) were available including 11,809 stroke patients and 34,853 control subjects: 11 of them had subgroups about stroke (CE stroke and non-CE stroke) and one cohort just discussed the relation to non-CE stroke (supplementary Table 2). Figure 3 respectively presented the association among rs10033464 and overall stroke, CE stroke, non-CE stroke. Obviously, Fig. 3a showed no association was found between rs10033464 and overall stroke (OR 1.04, $95 \%$ CI 0.97-1.10) without heterogeneity ( $Q=7.13$, $\mathrm{I}^{2}=0.0 \%$ ). However, subgroup analysis demonstrated that rs10033464 was significantly associated with $\mathrm{CE}$ stroke (OR 1.14, $95 \%$ CI 1.02-1.26) without heterogeneity $\left(\mathrm{Q}=7.58, \mathrm{I}^{2}=0.0 \%\right)$ (Fig. 3b). No association was found for non-CE stroke (OR 0.97, $95 \%$ CI 0.90-1.03) (Fig. 3c).

\section{Publication bias and sensitivity analysis}

We further investigated the publication bias of every metaanalysis about rs2200733 and rs10033464 using Begg test and funnel plot (supplementary Fig. 1a-f). There was no evidence of publication bias (all $P>0.05$ ). The sensitivity analysis also illuminated that none of including studies or cohorts influenced the stability of pooled ORs (supplementary Fig. 2a-f).

\section{Discussion}

The two SNPs (rs2200733, rs10033464) on chromosome $4 q 25$ were first found to be strongly associated with atrial fibrillation (AF) in European descent by Gudbjartsson et al. [19]. Another GWAS study verified these two variants also contributed to ischemic stoke, especially cardioembolic stroke [7]. However, the mechanism whereby the genetic variants exert their same effects on these two related phenotypes remains to be elucidated. The most possible explanation might be that the $4 \mathrm{q} 25$ locus associates with $\mathrm{AF}$ and therefore indirectly also associates with $\mathrm{CE}$ stroke.

We carried out a case-control study and failed to replicate the relevance of two SNPs and stroke in Chinese Han population. This discrepancy may be explained by the following reasons: (1) cardioembolic stroke as well as stroke patients with AF were excluded from our study; (2) these strongest signals may reflect genuine susceptibility effects on cardioembolic stroke but not other subtypes; (3) due to small sample size, we may lack enough power to detect the associations between these two SNPs and stroke. (4) genetic heterogeneity in different population. In China, among all subtypes of stroke, intracerebral hemorrhage accounts for $20-40 \%$ of strokes in the Chinese population. In contrast, the majority (80-90\%) of strokes in most western populations are cerebral infarctions [20]. The reasons for the high incidence of stroke, especially the hemorrhagic subtype, among the Chinese population, indicating different genetic background are more implicated in the pathogenesis of disease development. Furthermore, the distributions of these two SNPs were different between various ethnic populations (for example, the frequency of $\mathrm{T}$ allele for rs2200733 in Chinese population is 0.537 compared to 0.104 in European cohorts based on Hapmap database) and there was populationspecific genetic effect as a result of gene-gene and geneenvironment interactions.

Since the first description of the association between $4 q 25$ and stroke, a number of replication studies with conflicting results appeared. To date, there still was not final and coherent conclusion. Hence, combining our own cohort, we performed a meta-analysis for SNPs rs2200733 and rs10033464, respectively. Our meta-analysis harbored impressively large sample size from different studies, so the results seemed to be more reliable and stable than those from single study. However, there are limitations in it: (1) All included literatures were published in English or Chinese, therefore, we might lose some articles meeting our inclusion criteria but issued in other languages. (2) We only recruited relevant papers which had been published, so it was possible to miss some not published and inevitably to cause publication bias. (3) The studies included in our meta-analysis were all case-control studies or population-based studies, which precluded the further comments on cause-effect relationship. (4) Although we respectively carried out meta-analysis in $\mathrm{CE}$ stroke subgroup and non-CE stroke subgroup, not all included associated literatures had the data about subgroups. To some extent, the meta-analysis results of non-CE stroke subgroup were likely influenced by phenotypic misclassification. In view of this, it seemed plausible that our result that rs2200733 marginally associated with non-CE stroke susceptibility was not exactly true. The relevance between rs2200733 and overall stroke was also affected by CE stroke. So, we seem to conclude that rs2200733 only led to CE stroke. (5) The population in our meta-analysis most was from Europe and we failed to find any evidence to report the association between these two SNPs and cardioembolic stroke in Chinese Han population after 
extensively searching the literatures, thus studies undergoing in other countries or places (especially in China) should be needed. (6) due to no association between these two SNPs and AF were determinate in present study, we did not know whether the association remained after excluding the $\mathrm{CE}$ stroke patients with $\mathrm{AF}$.

\section{Conclusion}

In conclusion, by combing all available data from genetic studies on 4q25 and stroke, we confirmed rs2200733 and rs 10033464 both were associated with CE stroke. However, the molecular basis under it still needs to be elucidated and well-designed studies with a large number of subjects from different countries and regions should be conducted in the future.

Acknowledgments This work was supported by grants from National "973" projects (Nos. 2012CB518004 and 2012CB517801), "863" project (No. 2012AA02A510), Ministry of Education of China for Outstanding Young Teachers in University (20110142120012) and Key Project of Health Ministration. We would like to thank my collaborators for their efforts in recruiting patients and controls for this study. We want to especially acknowledge all the participants in this study.

Conflict of interest The authors declare that they have no conflict of interest.

Open Access This article is distributed under the terms of the Creative Commons Attribution License which permits any use, distribution, and reproduction in any medium, provided the original author(s) and the source are credited.

\section{References}

1. Strong K, Mathers C, Bonita R (2007) Preventing stroke: saving lives around the world. Lancet Neurol 6:182-187

2. Zhao D, Liu J, Wang W, Zeng Z, Cheng J, Sun J, Wu Z (2008) Epidemiological transition of stroke in China: twenty-one-year observational study from the Sino-MONICA-Beijing Project. Stroke 39(6): 1668-1674

3. Hankey GJ (2006) Potential new risk factors for ischemic stroke: what is their potential? Stroke 37:2181-2188

4. Stankovic S, Majkic-Singh N (2010) Genetic aspects of ischemic stroke: coagulation, homocysteine, and lipoprotein metabolism as potential risk factors. Crit Rev Clin Lab Sci 47:72-123

5. Bak S, Gaist D, Sindrup SH, Skytthe A, Christensen K (2002) Genetic liability in stroke: a long-term follow-up study of Danish twins. Stroke 33(3):769-774
6. Liao D, Myers R, Hunt S, Shahar E, Paton C et al (1997) Familial history of stroke and stroke risk. The Family Heart Study. Stroke 28:1908-1912

7. Gretarsdottir S, Thorleifsson G, Manolescu A, Styrkarsdottir U, Helgadottir A et al (2008) Risk variants for atrial fibrillation on chromosome $4 \mathrm{q} 25$ associate with ischemic stroke. Ann Neurol 64:402-409

8. Bellenguez C, Bevan S, Gschwendtner A, Spencer CC, Burgess AI et al (2012) Genome-wide association study identifies a variant in HDAC9 associated with large vessel ischemic stroke. Nat Genet 44:328-333

9. Carty CL, Buzkova P, Fornage M, Franceschini N, Cole S et al (2012) Associations between incident ischemic stroke events and stroke and cardiovascular disease-related genome-wide association studies single nucleotide polymorphisms in the population architecture using genomics and epidemiology study. Circ Cardiovasc Genet 5:210-216

10. Ikram MA, Seshadri S, Bis JC, Fornage M, DeStefano AL et al (2009) Genomewide association studies of stroke. N Engl J Med 360:1718-1728

11. Lemmens R, Buysschaert I, Geelen V, Fernandez-Cadenas I, Montaner J et al (2010) The association of the 4q25 susceptibility variant for atrial fibrillation with stroke is limited to stroke of cardioembolic etiology. Stroke 41:1850-1857

12. Shi L, Li C, Wang C, Xia Y, Wu G et al (2009) Assessment of association of rs 2200733 on chromosome 4q25 with atrial fibrillation and ischemic stroke in a Chinese Han population. Hum Genet 126:843-849

13. Traylor M, Farrall M, Holliday EG, Sudlow C, Hopewell JC et al (2012) Genetic risk factors for ischaemic stroke and its subtypes (the METASTROKE Collaboration): a meta-analysis of genomewide association studies. Lancet Neurol 11:951-962

14. Virani SS, Brautbar A, Lee VV, Elayda M, Sami S et al (2011) Usefulness of single nucleotide polymorphism in chromosome $4 \mathrm{q} 25$ to predict in-hospital and long-term development of atrial fibrillation and survival in patients undergoing coronary artery bypass grafting. Am J Cardiol 107:1504-1509

15. Wnuk M, Pera J, Jagiella J, Szczygiel E, Ferens A et al (2011) The rs2200733 variant on chromosome $4 \mathrm{q} 25$ is a risk factor for cardioembolic stroke related to atrial fibrillation in Polish patients. Neurol Neurochir Pol 45:148-152

16. Ding H, Wu B, Wang H, Lu Z, Yan J et al (2010) A novel loss-offunction DDAH1 promoter polymorphism is associated with increased susceptibility to thrombosis stroke and coronary heart disease. Circ Res 106:1145-1152

17. Ding H, Cui G, Zhang L, Xu Y, Bao X et al (2010) Association of common variants of CYP4A11 and CYP4F2 with stroke in the Han Chinese population. Pharmacogenet Genomics 20:187-194

18. Higgins JP, Thompson SG, Deeks JJ, Altman DG (2003) Measuring inconsistency in meta-analyses. BMJ 327:557-560

19. Gudbjartsson DF, Arnar DO, Helgadottir A, Gretarsdottir S, Holm $\mathrm{H}$ et al (2007) Variants conferring risk of atrial fibrillation on chromosome 4q25. Nature 448:353-357

20. Reed DM (1990) The paradox of high risk of stroke in populations with low risk of coronary heart disease. Am J Epidemiol 131:579-588 